\title{
Knock-down of P-glycoprotein reverses taxol resistance in ovarian cancer multicellular spheroids
}

\author{
HUI XING ${ }^{1,2^{*}}$, SHIXUAN WANG ${ }^{1 *}$, DANHUI WENG ${ }^{1}$, GANG CHEN $^{1}$, XIAOKUI YANG ${ }^{1}$, \\ JIANFENG ZHOU ${ }^{1}$, GANG XU ${ }^{1}$, YUNPIN LU ${ }^{1}$ and DING MA ${ }^{1}$ \\ ${ }^{1}$ Cancer Biology Research Center, Tongji Hospita, Tongji Medical College, Huazhong University \\ of Science and Technology, Wuhan, Hubei 430030; ${ }^{2}$ Department of Obsterics and Gynecology, Xiangfan Hospital, \\ Tongji Medical College, Huazhong University of Science and Technology, Xiangfan, Hubei 441021, P.R. China
}

Received August 16, 2006; Accepted September 18, 2006

\begin{abstract}
We previously established that multicellular ovarian cancer spheroids develop intrinsic multidrug resistance with the appearance of quiescent cell areas. p27 protein is a determinant of such resistance. However, the precise molecular basis of such resistance remains unknown. We demonstrated herein that these multicellular ovarian cancer spheroids expressed high levels of p27 and P-gp protein. Compared with monolayer cells, there is a significant increase in the resistance of spheroids cells to anticancer reagent Taxol. Antisense oligodeoxynucleotide not only mediated downregulation of $\mathrm{p} 27$, but also $\mathrm{P}$-gp expression in multicellular spheroids. Selective small interfering RNAs (siRNA) of P-gp with MDR1-targeted short hairpin RNAs (shRNA) expression vector sensitized the cells to Taxol. These results suggest that both p27 and P-gp can modulate Taxol sensitivity respectively, while p27 requires P-gp for its full function. Increased P-gp protein expression through p27 mediation is one of the major mechanisms of Taxol resistance in ovarian cancer multicellular spheroids.
\end{abstract}

\section{Introduction}

Although chemotherapy remains a major treatment modality for human ovarian cancer, chemoresistance is a clinical problem that severely limits treatment success. It is now widely accepted that development of P-gp-mediated drug resistance in tumors is a major barrier for the effectiveness

Correspondence to: Dr Ding Ma, Cancer Biology Research Center, Tongji Hospital, Tongji Medical College, Huazhong University of Science and Technology, 1095 Jiefang Ave., Wuhan, Hubei 430030, P.R. China

E-mail:dma@tjh.tjmu.edu.cn; dingma424@yahoo.com

\section{${ }^{*}$ Contributed equally}

Key words: multicellular spheroids, P-gp, taxol drug resistance shRNA of cancer therapy (1). P-glycoprotein (P-gp) is a $150-180 \mathrm{kDa}$ membrane phosphoglycoprotein encoded by the multidrug resistance (MDR1) gene, which functions as an energydependent drug transporter with broad specificity.

The regulation of $\mathrm{P}$-gp expression has been widely investigated to better understand its pathophysiological implications. In particular, it has been shown that P-gp expression is regulated through transcriptional and posttranscriptional mechanisms and by various endogenous and environmental stimuli that evoke stress response (2). Such intrinsic drug resistance can be recapitulated in vitro by growing tumor cells as multicellular spheroids (MCS) that exhibit many of the properties of solid tumors. In contrast to monolayer cell culture, multicellular spheroids display a reduced growth fraction and resistance to a wide variety of cytotoxic agents (3-5). Numerous studies have shown that intrinsic drug resistance is expressed in the areas of quiescent cells in large prostate tumor spheroids, but not in small shperiods containing proliferating cells $(1,6)$, indicating that the cell cycle arrest may induce P-gp expression. It has been shown that up-regulation of $\mathrm{P}$-gp in $\mathrm{G} 0 / \mathrm{G} 1$ phase cells requires coexpression of p27 but not p21 $(5,7)$. p27 is a member of cyclin-dependent kinase inhibitors. By inhibiting a variety of cyclin-Cdk complexes, p27 regulates progression from G1 to $\mathrm{S}$ phase $(8,9)$. p27 is up-regulated by cell-cell contact in normal cell culture $(3,10-12)$. Our previous study demonstrated that multicellular ovarian cancer spheroids develop intrinsic multidrug resistance with the appearance of quiescent cell areas. p27 protein is a determinant of such resistance and that down-regulation of p27 sensitizes the cells to Taxol. Furthermore, p27 up-regulates the P-gp protein expression in multicellular ovarian cancer spheroids cells (13). Collectively, these data suggest that p27-mediated chemoresistance may be caused in part by the up-regulation of P-gp protein. To confirm this hypothesis, we studied P-gp expression in ovarian cancer cells cultured in a threedimensional context.

Targeted gene silencing in mammalian cells by RNA interference (RNAi) using small interfering RNAs (siRNAs) was described by Elbashir et al (14). A number of groups have reported a solution based on transcription of short hairpin RNAs (shRNAs) by RNA polymerase III (pol III) $(15,16)$. The 
hairpins of these short RNAs are processed to generate siRNAs and induce gene silencing. Transfection of plasmids with pol III promoters driving hairpin RNAs can eliminate the expression of a target gene.

In this study, we used MDR1-targeted shRNA transfection to knock-down the P-gp expression and tested whether the decreased sensitivity of the multicellular spheroid cells to Taxol was due to down-regulation of P-gp.

\section{Materials and methods}

Cell lines. The human ovarian cancer cell lines, A2780, CAOV3, SKOV3 and SW626 cells, were purchased from American Type Culture Collection (ATCC). The cells were grown according to the vendor's instructions. Briefly, cells were cultured in $150-\mathrm{mm}$ culture bottles at $37^{\circ} \mathrm{C}$ in an atmosphere of $5 \% \mathrm{CO}_{2}$ in RPMI-1640 (A2780), DMEM (CAOV3 and SW626), or McCoy's 5A (SKOV3) containing $15 \%$ FBS. Multi-cellular spheroids were prepared by a liquid overlay method as described previously (14).

Reagents and antibodies. Trizol was purchased from Gibco LifeScience Co., CA, USA. Lipofectamine 2000 was obtained from Invitrogen, San Diego, CA, USA. Taxol were purchased from Bristol-Myers Squibb Co., Princeton, USA. Mouse monoclonal anti-human p27 and P-gp were purchased from Neomarkers Co., Frement, USA. The nucleotide sequences of antisense (ASON) and sense (SON) p27 were: 5'-CAT CTT CCC GGG TCT GCA CGA CCG CC-3' and 5'-CAG CAT CTG ACC TCG CCT TGG CTC TCG CC-3', respectively (17). They were purchased from Shanghai Biotechnology Co. Ltd., Shanghai, China. Every base was modified by sulphur.

Plasmids. The shRNA linearized vector pSIREN carrying the human U6 shRNA promoters was purchased from Biosciences Clontech, Franklin, USA. The oligonucleotides encoding the human MDR1 shRNA and flanking with BamHI and EcoR1 ends were: 5'-GATCCCATTCGCTATGGC CGTGAATTCAAGAGATTCACGGCCATAGCGAAT GTTTTTTG-3' and 5'-GCTTTTCCAAAAAACATTCG CTATGGCCGTGAATCTCTTGAATTCACGGCCATA GCGACTTAA-3'. Searches of the human genome database (BLAST) were carried out to ensure the sequences would not target other gene transcripts. These oligonucleotides were annealed and cloned into pSIREN following its restriction with BamHI and EcoR1. In addition, the negative control annealed oligonucleotide (Biosciences Clontech) was also cloned into pSIREN, termed negative control vector.

Transfections. The oligo/Lipofectamine complex was diluted to $1 \mathrm{X}$ concentration and overlaid onto cells. After $12 \mathrm{~h}$ of incubation, cells were rinsed with phosphate-buffered saline (PBS), harvested with $0.25 \%$ trypsin, and suspended in complete medium containing $10 \%$ FBS for various assays.

For RNAi targeting, the A2780 and CAOV3 cells cultured in 6-well plates were transfected with indicated plasmids using Lipofectamine 2000. After $6 \mathrm{~h}$ of incubation, the transfection solution was removed with fresh complete growth medium. The cells were used for detection of the expression of siRNAs $48 \mathrm{~h}$ post-transfection.

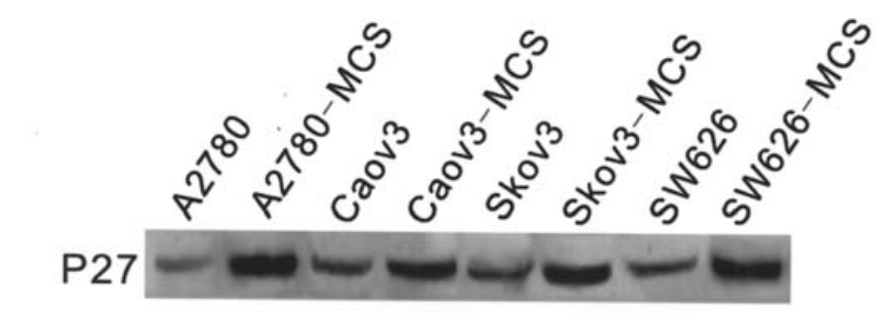

$\beta$-actin

Figure 1. Western blot analysis of p27 in a panel of human ovarian cancer cell lines. The expression levels of p27 in A2780, CAOV3, SKOV3, and SW626 ovarian cancer cells and their multicellular spheroids derived from a three-dimensional culture were analyzed by Western blot. When monolayer culture was replaced by three-dimensional culture, a constant up-regulation of p27 protein was observed in these cell lines. These results conform with the involvement of p27 in chemoresistance, as we previously reported.

Apoptotic analysis by flow cytometry. Cells were harvested with $0.25 \%$ trypsin and washed once with PBS. After centrifugation, cells were fixed in $80 \%$ ice-cold ethanol overnight at $-20^{\circ} \mathrm{C}$. Cells were then incubated in $500 \mu 1$ of the same buffer containing $50 \mu \mathrm{g} / \mathrm{ml}$ of propidium iodide (PI) and $1 \mathrm{mg} /$ $\mathrm{ml}$ of RNAase for $30 \mathrm{~min}$. The analysis of apoptotic cells was performed on a FACScan flow cytometer, and the data were analyzed using cell Fit software.

Western blot analysis. All cell samples were lysed with icecold NP-40 lysing buffer (1\% NP-40, 10\% glycerol, $20 \mathrm{mM}$ Tris- $\mathrm{HCl}$ at $\mathrm{pH} 7.5,137 \mathrm{mM} \mathrm{NaCl}, 1 \mathrm{mM}$ phenyimethyisulfonyl fluoride and $5 \mu \mathrm{g} / \mathrm{ml}$ of aprotinin). The soluble protein solutions were subjected to SDS-PAGE and transferred to a nitrocellulose membrane by electrophoresis in a semidry chamber, Biometra, Germany. P-gp and p-27 were identified by immunoblot analysis using anti-P-gp and anti-p27 antibody, respectively. Followed by incubation with HRP-conjugated secondary antibody at 1:5000 dilution for $1 \mathrm{~h}$ at $37^{\circ} \mathrm{C}$. Protein signals were detected by enhanced chemiluminesence (Pierce Rockford, USA).

$R T-P C R$. Total RNA was isolated from each group of cells using Trizol Regent according the manufacturer's instructions. Total cellular RNA was used for reverse transcription of cDNA by a standard technique. Obtained cDNA were amplified using specific primers: 5'-TAC AGT GGA ATT GGT GCT GG-3' (sense) and 5'-CTA ATT GCT GCC AAG ACC TC-3' (antisense) that amplify a 433-bp fragment of MDR1 cDNA. The primers for glyceraldehyde-3-phosphate dehydrogenase (GAPDH, internal control) were 5'-ACG GAT TTG GTC GTA TTG GG-3' (sense) and 5'-TGA TTT TGG AGG GAT CTC GC-3' (antisense) that amplify a 230-bp fragment of GAPDH cDNA. After pre-denaturation at $94^{\circ} \mathrm{C}$ for $5 \mathrm{~min}$, PCR were performed for 30 cycles: $30 \mathrm{sec}$ denaturation at $94^{\circ} \mathrm{C}$, followed by annealing for $1 \mathrm{~min}$ at $55^{\circ} \mathrm{C}$ and finally extension for $1 \mathrm{~min}$ at $72^{\circ} \mathrm{C}$.

Confocal laser scanning microscopy. Briefly, the cells were incubated with P-gp monoclonal antibodies (1:100 dilution) at room temperature for $45 \mathrm{~min}$ followed by incubation with fluorescein-conjugated goat anti-mouse $\operatorname{IgG}$ at $37^{\circ} \mathrm{C}$ for 


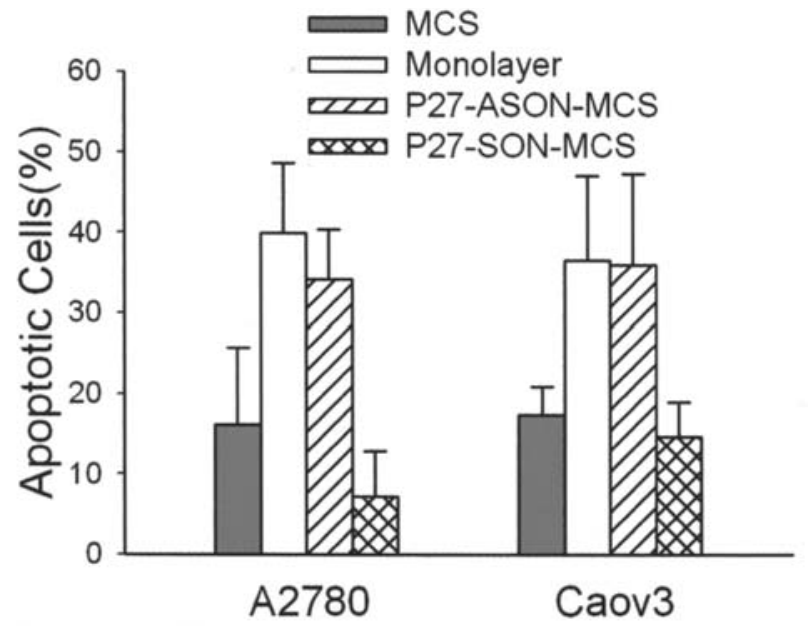

Figure 2. Apoptosis assay of A2780 and CAOV3 cells by flow cytometry. After transfection with the indicated DNA, the cells were plated into a three-dimensional culture for 3 days. Cells were then exposed to $20.0 \mu \mathrm{mol} / 1$ of Taxol for $24 \mathrm{~h}$. Hypodiploid (apoptotic) DNA content was evaluated by propidium iodide staining and flow cytometry. Compared to A2780 and CAOV3 monolayer cells, Taxol treatment of the spheroid cells significantly decreased apoptosis rate as defined by flow cytometry of DNA histogram using flow cytometry. The apoptosis rate was significantly higher in the spheroid cells treated with p27-ASON, but not with p27-SON. The data showed that spheroid cells were resistant to Taxol, and the antisense p27 oligonucleotides sensitized these cells to Taxol.

$30 \mathrm{~min}$. The cells were stained with PI solution for $20 \mathrm{~min}$ and analyzed under confocal laser scanning microscopy with a $488 \mathrm{~nm}$ argon-ion laser (LSCM, Leica, TCSSP, Germany).

Statistical analysis. All experiments were performed in triplicate. Student's t-test was used to evaluate the differences between two groups. SPSS 10.0 for Windows software analysis system was used for statistical analysis. Data are presented as mean $\pm \mathrm{SE}$ (standard error).

\section{Results}

Increased chemoresistance in ovarian cancer multicellular spheroids due to high expression levels of p27. To firm the basis on which our subsequent studies were carried out and to confirm our previous report that p27 is implicated in Taxol resistance in ovarian cancer multicellular spheroids, the relative levels of p27 expression among different ovarian cancer cell lines and multicellular spheroids were compared by using Western blot analysis. Fig. 1A shows the expression levels of p27 in a panel of human ovarian cancer cell lines and their multicellular spheroids derived from a three-dimensional culture. When monolayer culture was replaced by threedimensional culture, a constant up-regulation of p27 protein was observed in these cell lines. These results confirm the involvement of p27 in chemoresistance, as previously reported by our laboratory (14).

We used antisense oligonucleotide to affect p27 expression in these cells by using a p27-specific ASON, a 29-bp nucleotide sequence that is complementary to the 5'-untranslated region of the p27 mRNA. Consistent with previous observations, transfection of A2780 cells with $200 \mathrm{nM}$ of p27-specific ASON caused $70-80 \%$ reduction of $\mathrm{p} 27$
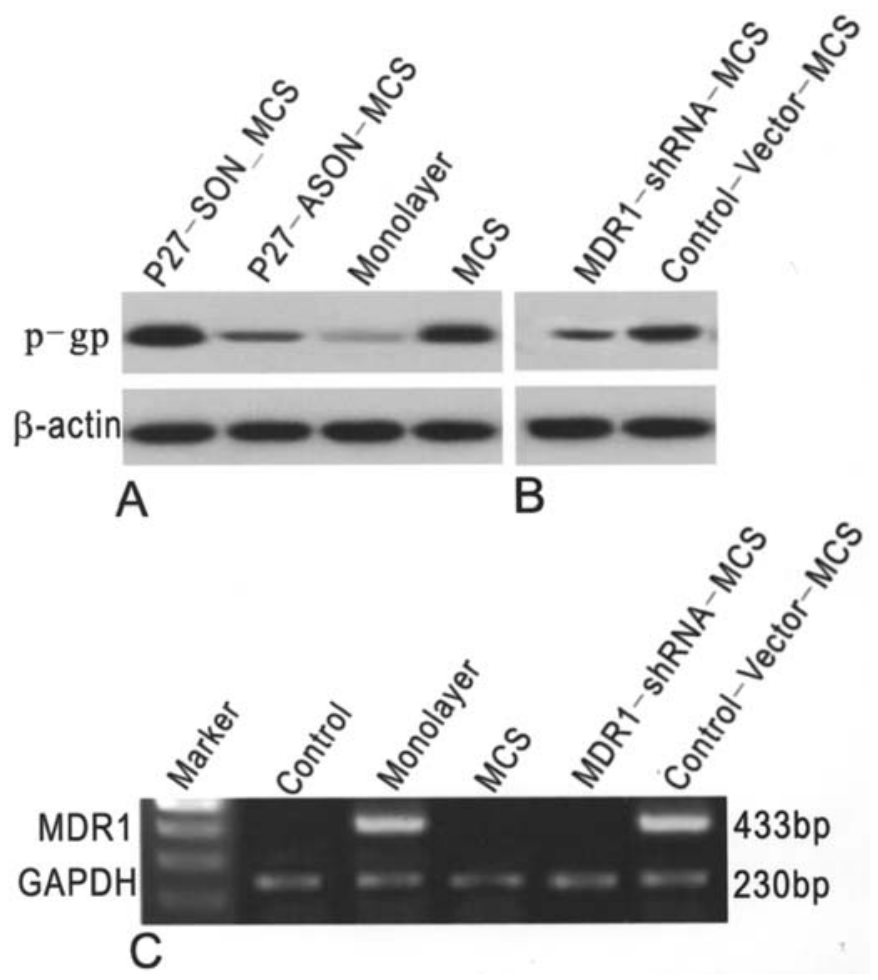

Figure 3. Detection of MDR1 from both protein and mRNA levels in A2780 cells by using Western blot analysis and RT-PCR, respectively. (A) P-gp protein is clearly expressed in multicellular spheroids (MCS) but is lower in monolayer cells (Monolayer). Treatment of antisense (ASON) but not sense (SON) p27 oligonucleotides significantly down-regulated P-gp protein levels in aggregates cells. These data demonstrate that, in ovarian cancer multicellular spheroids, the P-gp protein seems to be correlated with increased levels of p27, which mediates cell-cycle arrest in the G1 phase. After $48 \mathrm{~h}$ of transfection, the cells were plated into a three-dimensional culture for 3 days. Then the total mRNA and protein were retrieved and purified. (B) Compared with the results for control vector-transfected cells and non-transfected cells, introducing the MDR1 shRNA resulted in the loss of P-gp protein in multi-cellular spheroids. (C) Ethidiumbromide-stained agarose gel after RT-PCR amplification of MDR1 mRNA (433 bp). All PCRs were co-amplified with GADPH as an internal control (230 bp). DdH2O served as negative control. Multicellular spheroids had positive MDR1 mRNA expression (MCS). The MDR1-shRNA-MCS, but not Control-Vector-MCS resulted in the loss of MDR1 mRNA expression.

expression. In contrast, treatment of these cells with the same concentration of SON resulted in a slight decrease in p27 expression (data not shown).

To evaluate the effect of p27 on drug resistance of the ovarian cancer cells, the sensitivity of A2780 cell and their multicellular spheroids to Taxol were analyzed by flow cytometry.

As shown in Fig. 2, compared to A2780 and CAOV3 monolayer cells, the apoptotic rates of the spheroid cells treated with Taxol decreased significantly as defined by DNA histogram of flow cytometry. Furthermore, the apoptotic rates were significantly higher in the spheroid cells treated with p27-ASON, but not with p27-SON. The data suggested that p27 is a determinant of Taxol sensitivity in ovarian cancer spheroid cells, and the antisense p27 oligonucleotides sensitized these cells to Taxol.

Increased P-gp expression through p27 mediation reverses Taxol resistance in multicellular spheroids. Recent studies 

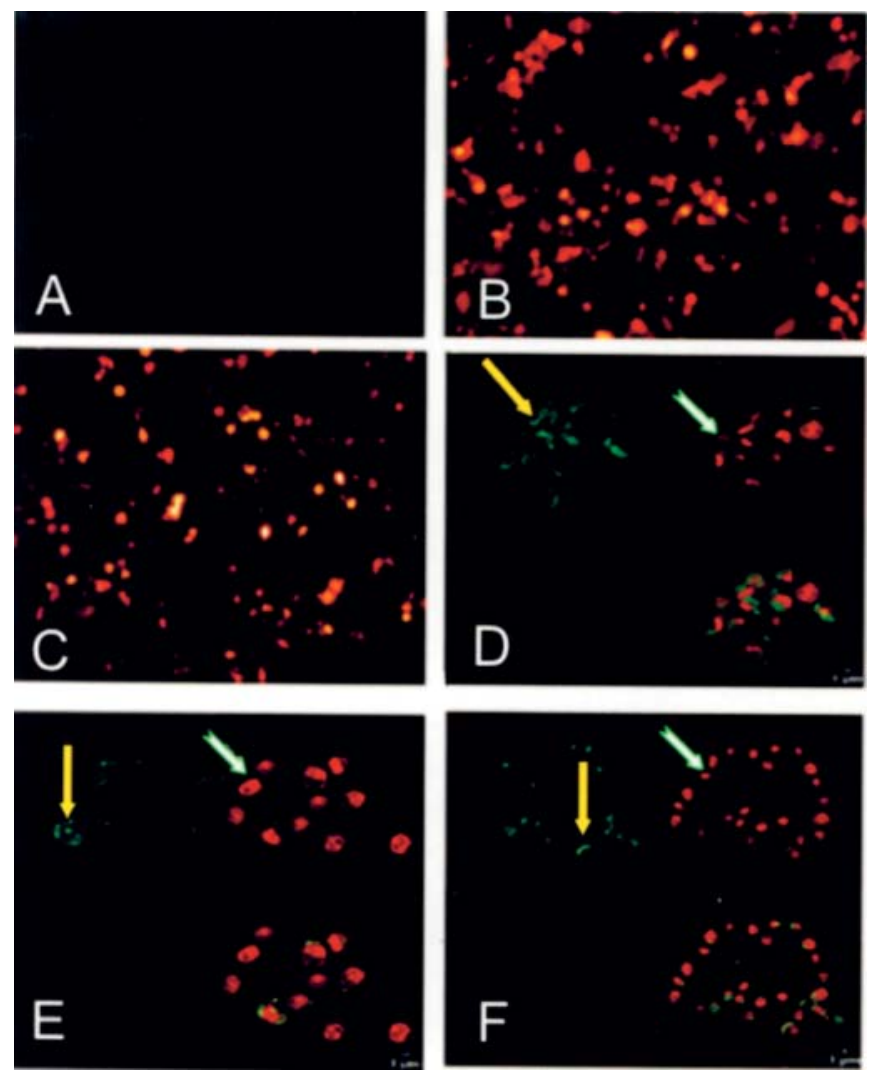

Figure 4. Fluorescent detection of MDR1-shRNA in A2780 cells. A2780 cells were transiently transfected with control-vector or MDR1-shRNA vector and cultured in growth medium for $48 \mathrm{~h}$. After a 48 -h infection the fluorescence was detected by using fluorescent microscope. The red fluorescence of luciferase illustrated the efficiency of the transfection. (A) A2780 cells (non-transfected), (B) MDR1-shRNA transfected cells, (C) negative-control-vector transfected cells. After a 48-h infection the cells were then plated into a three-dimensional culture for 3 days. The subcellular distribution of P-gp in human ovarian cancer T2780 cells was evaluated by immunocytochemistry using Confocal laser scanning microscopy as described in Materials and methods. The A2780 nuclei were stained as red by using PI (white-green arrows in D-F) and P-gp proteins were stained green (yellow arrows in D-F). Subcellular compartmentalization of P-gp in ovarian cancer A2780 cell is frequently observed in the cell membrane. Intense P-gp membrane staining was observed in multicellular spheroids (D). Decreased membrane staining of P-gp was found in MDR1-shRNA multicellular spheroid cells (E) but not in control-vector cells $(\mathrm{F})$.

suggest that up-regulation of P-gp in G0/G1 phase cells requires coexpression of p27 but not p21 $(5,7)$. We studied whether p27 expression level correlated with $\mathrm{P}$-gp protein expression. Western blot analysis revealed that expression of $\mathrm{P}-\mathrm{gp}$ protein was significantly decreased in aggregate cells treated with p27-ASON but not with p27-SON (Fig. 3A).

$\mathrm{P}$-gp expression has been implicated as playing an important role in Taxol resistance (18). In this study, we investigated whether P-gp protein expression correlated with Taxol resistance in multicellular spheroids cells. Western blot analysis revealed that $\mathrm{P}-\mathrm{gp}$ protein was expressed in multicellular spheroids (MCS), but was lower in monolayer cells (Fig. 3A).

These results suggest that increased P-gp protein expression by $\mathrm{p} 27$ is one of the major mechanisms underlying Taxol resistance in ovarian cancer multicellular spheroids, although
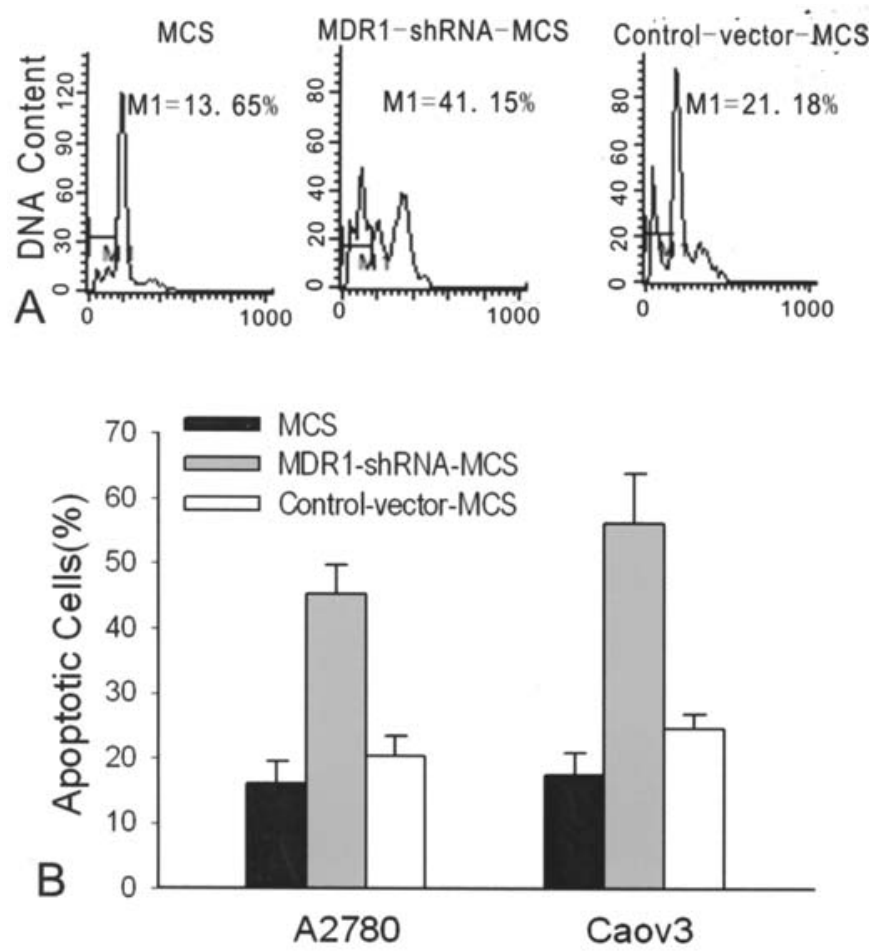

Figure 5. Effect of P-gp targeting on ovarian cancer cell apoptosis by flow cytometry. After $48 \mathrm{~h}$ of transfection, the cells were plated into a threedimensional culture for 3 days. Cells were then exposed to $20.0 \mu \mathrm{mol} / 1$ of Taxol for $24 \mathrm{~h}$. Hypodiploid (apoptotic) DNA content was evaluated by propidium iodide staining and flow cytometry. (A) One representative experiment in A2780 cells is shown. (B) Data represent means of percentage of apoptosis \pm SE of 3 independent experiments in A2780 and Caov3 cells. Compared with the results for the control vector transfected MCS cells and non-transfected MCS cells, the MDR1 shRNA MCS cells showed increased sensitivity to Taxol in both A2780 and CAOV3 cells.

other factors regulated by p27 might be involved in Taxol resistance.

High levels of P-gp is required for apoptotic resistance to Taxol in ovarian cancer multicellular spheroids. To further determine the role of P-gp in mediating the resistance of ovarian cancer multicellular spheroids to Taxol, we constructed an MDR1 transient shRNA expression vector, termed MDR1- shRNA vector, and a negative control vector. Both were transfected into A2780 and CAOV3 cells, respectively. Efficiency of shRNA transfection was detected by using fluorescence microscopy after $48 \mathrm{~h}$ of transfection (Fig. 4). The red fluorescence of luciferase illustrated the efficiency of the transfection.

To evaluate the silencing effects of MDR1-shRNA-vector quantitatively, the MDR1 expression was detected at both protein and mRNA level, by using Western blot analysis and RT-PCR, respectively (Fig. 3B and C). The subcellular distribution of P-gp in human ovrian cancer A2780 cells was illustrated by using Confocal laser scanning microscopy. As shown in Fig. 4D-F, subcellular compartmentalization of P-gp in ovarian cancer A2780 cell appeared frequently on the cell membrane. The intense membrane staining of P-gp was observed in multicellular spheroids (Fig. 4D). A decreased membrane staining of P-gp was found in MDR1-shRNA 
multicellular spheroid cells (Fig. 4E) but not in control-vector cells (Fig. 4F).

The results showed that the expression of $\mathrm{P}-\mathrm{gp}$ was definitely suppressed, compared with that of control vectortransfected cells or non-transfected cells. Introducing the MDR1 shRNA resulted in the loss of P-gp protein in multicellular spheroids. While mRNA level of MDR1 showed the same changes as compared with that of protein level.

We then compared the sensitivity of the MDR1 shRNA vector-transfected A2780 and CAOV3 MCS cells (MDR1shRNA-MCS) with that of the control vector-transfected MCS (control-vector-MCS) cells and non-transfected MCS cells to Taxol. As shown in Fig. 5, compared with the results for the control vector transfected MCS cells and non-transfected MCS cells, the MDR1 shRNA MCS cells showed increased sensitivity to Taxol in both A2780 and CAOV3 cells. These data demonstrated that $\mathrm{p} 27$ requires P-gp for its full function. Increased $\mathrm{P}-\mathrm{gp}$ protein expression is one of the major mechanisms underlying taxol resistance in multicellular spheroid cells.

\section{Discussion}

Ovarian epithelial cancer is the fifth leading cause of death among female cancers in China. Although chemotherapeutic agents, such as Taxol, are widely used for the treatment of ovarian cancer, chemoresistance remains a major therapeutic problem, and the molecular mechanisms leading to chemoresistance are still poorly understood.

Traditionally, the mechanisms of drug resistance have been identified and functionally characterized in unicellular models. Implicit in these unicellular models is the lack of consideration of host-tumor cell interactions that may participate in the emergency of the drug-resistant phenotype. We propose that the initial selection pressure for tumor growth and survival is represented by host-tumor cell interactions and that these same interactions may participate in modulating drug response and emergence of a stable drug resistance. The data of Hazlehurst et al (19) indicated the importance of the microenvironment in mediating the expression of a functional drug resistance phenotype. Thus, we suggest that in order to identify clinically relevant drug-resistant targets, new models must be developed for reconstitution of host-tumor cell interactions. In the present study, we used one such model, namely multicellular spheroids as a model to investigate the survival effects of cell adhesion. This model more closely resembles the architecture of a solid tumor in vivo.

We previously established that cells in compact spheroids with high expression of p27 have increased cells of G0/G1 phase, but reduced cells of $\mathrm{S}$ and $\mathrm{G} 2 / \mathrm{M}$ phases. After exposure to Taxol, aggregate cells in $\mathrm{S}$ and $\mathrm{G} 2 / \mathrm{M}$ phases tend to survive Taxol toxicity. In addition, we found that p27 was upregulated in parallel to the increased expression of P-gp in multicellular spheroids, which coincided with decreased tumor growth kinetics. We found that down-regulation of p27 by p27-ASON led to a clear increase in the proportion of A2780 and CAOV3 cells of S/G2 phase, reduced intercellular adhesion, and decreased expression of P-gp. All the above sensitized the ovarian cancer cells to the chemotherapeutic agent, Taxol. Therefore, sense p27 may be viewed as a drug resistance-associated protein. However, the precise relationships among p27, P-gp and drug resistance are worth further investigation.

In the present study, we found that cells in compact spheroids have a high expression of p27. In addition, p27 is up-regulated in parallel to the increased expression of P-gp in multicellular spheroids, which coincides with declined tumor growth kinetics. Our results suggest that increased P-gp protein expression by p27 is one of the major mechanisms underlying Taxol resistance in ovarian cancer multicellular spheroids, although other factors regulated by p27 might participate in Taxol resistance.

RNA interference (RNAi) is now established as a general method to silence gene expression in a variety of organisms $(14,20)$. When introduced into cells, dsRNA will interfere with the expression of homologous genes, subsequently disrupting their normal function. This suppression is mediated by 19-23-nt siRNAs, which induce degradation of mRNA based on complementary base pairing. All of these shortcomings of siRNA will be solved by the cellular expression of shRNA from vectors. shRNAs are expressed from mammalian promoters on DNA vectors which are introduced to cells by transfection or infection and possess double-stranded stems $\sim 19-23 \mathrm{nt}$ in length that will serve as substrates for Dicer $(21,22)$, and then effectively inhibit gene expression in a sequence-specific manner (23). In this study, we constructed a vector encoding shRNA targeting human MDR1 gene expression. The results showed that in A2780 and CAOV3 cells our MDR1-shRNA vectors reduced the expression of human MDR1 both in mRNA and protein level, when compared with the negative control. We then compared the sensitivity of the cells to Taxol. When compared with the results of the control vector transfected MCS cells and nontransfected MCS cells, the MDR1 shRNA MCS cells showed increased sensitivity to Taxol.

In summary, our studies demonstrate that p27 and P-gp may play an important role in protecting human ovarian cancer cells aganist Taxol-mediated cytotoxicity in three-dimentional culture. The increased P-gp protein expression regulated by $\mathrm{p} 27$ is one of the major mechanisms underlying Taxol resistance in MCS cells.

\section{Acknowledgements}

This work was supported by grants from the National Science Foundation of China (Nos. 30528012, 30571950) and '973' Program of China (No. 2002CB513100).

\section{References}

1. Martin C, Walker J, Rothnie A and Callaghan R: The expression of P-glycoprotein does influence the distribution of novel fluorescent compounds in solid tumour models. Br J Cancer 89: 1581-1589, 2003.

2. Yague E, Armesilla AL, Harrison G, Elliott J, Sardini A, Higgins CF and Raguz S, et al: P-glycoprotein (MDR1) expression in leukemic cells is regulated at two distinct steps, mRNA stabilization and translational initiation. J Biol Chem 278: 10344-10352, 2003.

3. St. Croix B, Florenes VA, Rak JW, Flanagan M, Bhattacharya N, Silingerland JM and Kerbel RS: Impact of the cyclin-dependent kinase inhibitor p27 on resistance of tumor cells to anti-cancer agents. Nature Med 2: 1204-1210, 1996. 
4. Sutherland LaRue KE, Khalil M and Freyer JP: Microenvironmental regulation of proliferation in multicellular spheroids is mediated through differential expression of cyclindependent kinase inhibitors. Cancer Res 64: 1621-1631, 2004.

5. Walker J, Martin C and Callaghan R: Inhibition of P-glycoprotein function by XR9576 in a solid tumour model can restore anticancer drug efficacy. Eur J Cancer 40: 594-605, 2004.

6. Wartenberg M, Fischer K, Hescheler J and Sauer H: Redox regulation of P-glycoprotein-mediated multi-drug resistance in multicellular prostate tumor spheroids. Int J Cancer 85: 267-274, 2000.

7. Wartenberg M, Fischer K, Hescheler J and Sauer H: Modulation of intrinsic P-glycoprotein expression in multicellular prostate tumor spheroids by cell cycle inhibitors. Biochim Biophys Acta 1589: 49-62, 2002.

8. Sherr CJ and Roberts JM: CDK inhibitors: positive and negative regulators of G1-phase progression. Genes Dev 13: 1501-1512, 1999.

9. Jirstrom K, Ringberg A, Ferno M, Anagnostaki L and Landerg G: Tissue microarray analyses of G1/S-regulatory proteins in ductal carcinoma in situ of the breast indicate that low cyclin D1 is associated with local recurrence. Br J Cancer 89: 1920-1926, 2003.

10. Mengst L, Dulic V, Slingerland JM, Lees E and Reed SI: A cell cycle-regulated inhibitor of cyclin-dependent kinase. Proc Natl Acad Sci USA 91: 5291-5295, 1994.

11. Polyak K, Kato J, Solomon MJ, Sherr CJ, Massague J, Roberts JM and Koff A: p27kip1, a cyclin-Cdk inhibitor, links transforming growth factor- $\beta$ and contact inhibition to cell cycle arrest. Genes Dev 8: 9-22, 1994.

12. Shiohara M: Absence of WAF1 mutations in a variety of human malignancies. Blood 84: 3781-3784, 1994.

13. Xing H, Wang S, Hu K, Tao W, Li J, Gao Q and Yang X, et al: Effect of the cyclin-dependent kinases inhibitor p27 on resistance of ovarian cancer multicellular spheroids to anticancer chemotherapy. J Cancer Res Clin Oncol 131: 511-519, 2005.
14. Elbashir SM, Harborth J, Lendeckel W, Yalcin A, Weber K and Tuschl T: Duplexes of 21-nucleotide RNAs mediate RNA interference in cultured mammalian cells. Nature Med 411: 494-498, 2001.

15. Brummelkamp TR, Bernards R and Agami R: A system for stable expression of short interfering RNAs in mammalian cells. Science 296: 550-553, 2002.

16. Paul CP, Good PD, Winer I and Engelke DR: Effective expression of small interfering RNA in human cells. Nat Biotechnol 20: 505-508, 2002.

17. Achenbach TV, Muller R and Slater EP: Synergistic antitumor effect of chemotherapy and antisense-mediated ablation of the cell cycle inhibitor p27. Clin Cancer Res 6: 3006-3014, 2000.

18. Duan Z, Brakora KA and Seiden MV: Inhibition of ABCB1 (MDR1) and ABCB4 (MDR3) expression by small interfering RNA and reversal of paclitaxel resistance in human ovarian cancer cells. Mol Cancer Ther 3: 833-838, 2004.

19. Hazlehurst LA, Landowski TH and Dalton WS: Role of the tumor microenvironment in mediating de novo resistance to drugs and physiological mediators of cell death. Oncogene 2: 7396-7402, 2003.

20. Couzin J: Breakthrough of the year: small RNAs make big splash. Science 28: 2296-2297, 2002.

21. Hasuwa H, Kaseda K, Einarsdottir T and Okabe M: Small interfering RNA and gene silencing in transgenic mice and rats. FEBS Lett 532: 227-230, 2002

22. Hemann MT, Fridman JS, Zilfou JT, Hernando E, Paddison PJ, Cordon Cardo C and Hannon GJ: An epiallelic series of p53 hypomorphs created by stable RNAi produces distinct tumor phenotypes in vivo. Nat Genet 33: 396-400, 2003.

23. Liu Xd, Shum MM, Liu Y, Liu Sz and Alec S: Short hairpin RNA and retroviral vector-mediated silencing of p53 in mammalian cells. Biochem Biophys Res Commun 324: 1173-1178, 2004. 